\title{
Evaluadores 2021
}

La Revista de la Asociación Argentina de Ortopedia y Traumatología agradece a los siguientes evaluadores su importante participación en la revisión de los trabajos recibidos, y espera seguir contando con su valiosa colaboración.

\author{
Albergo, José Ignacio \\ Hospital Italiano de Buenos Aires, Ciudad Autónoma de Buenos Aires, Argentina \\ Alfano, Federico \\ Obra Social del Personal de Dirección de Sanidad “Luis Pasteur”, Ciudad Autónoma de Buenos Aires, Argentina \\ Allende, Bartolomé Luis \\ Sanatorio Allende, Córdoba, Argentina \\ Alvarado Gómez, Fernando \\ Fundación Santa Fe de Bogotá, Colombia \\ Amadei, Rafael \\ Argüelles, Santiago \\ Autorino, Carlos M. \\ Ávila, Adriana \\ Bazán, Pedro L. \\ Berardinelli, Daniel \\ Bidolegui, Fernando M. \\ Bittelman, Sacha \\ Boretto, Jorge G. \\ Cabrera, Marcos N. \\ Cadena, Alejandro \\ Cagnone, Juan Carlos \\ Capomassi, Miguel \\ Carabelli, Guido \\ Caruso, Juan \\ Castellini, Jorge \\ Ceresetto, José Manuel \\ Chahin, Andrés \\ Cima, José Ignacio \\ Cinat, Gabriela \\ Clembosky, Gabriel \\ Costa Paz, Matías \\ Daroda, Sergio \\ D’ Elia, Martín \\ De Elias, Manuel \\ Delgado, Pedro J. \\ Della Rosa, Luciano \\ Denari, Ricardo G. \\ Hospital Municipal de Vicente López “Bernardo Houssay”, Buenos Aires, Argentina \\ Hospital Universitario Austral, Pilar, Buenos Aires, Argentina \\ Hospital de Ortopedia Para Niños "Dr. Germán Díaz Lombardo”, Ciudad de México, CDMX, México \\ Hospital Interzonal General de Agudos “Gral. José de San Martín”, La Plata, Buenos Aires, Argentina \\ Sanatorio Rivadavia, San Miguel de Tucumán, Tucumán, Argentina \\ Hospital Sirio Libanés, Ciudad Autónoma de Buenos Aires, Argentina \\ Instituto Seguridad del Trabajo, Viña del Mar, Chile \\ Hospital Italiano de Buenos Aires, Ciudad Autónoma de Buenos Aires, Argentina \\ Sanatorio Güemes, Ciudad Autónoma de Buenos Aires, Argentina \\ Hospital General de Agudos “Carlos G. Durand”, Ciudad Autónoma de Buenos Aires, Argentina \\ Asociación Argentina de Cirugía de la Mano y Reconstructiva del Miembro Superior, Argentina \\ Instituto Dr. Jaime Slullitel, Rosario, Santa Fe, Argentina \\ Hospital Italiano de Buenos Aires, Ciudad Autónoma de Buenos Aires, Argentina \\ Hospital Militar Central “Cirujano Mayor Dr. Cosme Argerich”, Ciudad Autónoma de Buenos Aires, Argentina \\ Swiss Medical Group, Ciudad Autónoma de Buenos Aires, Argentina \\ Hospital Británico de Buenos Aires, Ciudad Autónoma de Buenos Aires, Argentina \\ Clínica Las Condes, Santiago, Chile \\ Clínica Privada Vélez Sarsfield, Córdoba, Argentina \\ Instituto de Oncología “Dr. Ángel H. Roffo”, Universidad Nacional de Buenos Aires, Argentina \\ Hospital Británico de Buenos Aires, Ciudad Autónoma de Buenos Aires, Argentina \\ Hospital Italiano de Buenos Aires, Ciudad Autónoma de Buenos Aires, Argentina \\ GAMMA - Clínica de la Mano, Hombro y Codo, La Plata, Buenos Aires, Argentina \\ Hospital de Niños "Víctor J. Vilela", Rosario, Santa Fe, Argentina \\ Hospital Universitario Austral, Pilar, Buenos Aires, Argentina \\ Hospital Universitario HM Montepríncipe, Madrid, España \\ Hospital Interzonal General de Agudos “Eva Perón” (Ex Castex), Buenos Aires, Argentina \\ Hospital General de Agudos “Carlos G. Durand”, Ciudad Autónoma de Buenos Aires, Argentina
}


Díaz-Borjón, Efraín

Dohmen, Aurelia L.

Dorman, Guido

Escudero Sepúlveda, Andrés F.

Fernández, Claudio A.

Fernández Comber, Sergio

Ferraris, Luis

Galaretto, Germán

Gallucci, Gerardo

Garabano, Germán

García Rey, Eduardo

Gelosi, Jorge Federico

Girardi, Federico P.

Graieb, Ariel

Gruenberg, Marcelo

Guyot, Juan Pablo

Innocenti, Sergio

Jorba, Pedro

Jorge, Fernando D.

Korin, Jorge

Lanari Subiaur, Felipe

Lencina, Omar

López Millán, Lucas M.

Lopreite, Fernando A.

Lores, Carlos A.

Lucero, Carlos M.

Lucioni, María C.

Maletti, Pablo

Mangupli, Martín M.

Manzone, Patricio

Martínez, Carlos E.

Martínez, Saúl

Martínez, Solange

Masaragian, Héctor

Masquijo, Julio Javier

Mizdraji, Luciano

Monteagudo de la Rosa, Manuel

Moya, Daniel

Nacinovich, Francisco

Oñativia, José I.

Otero García, Álvaro
Hospital Ángeles Lomas, Hacienda de las Palmas, México

Hospital Interzonal General de Agudos "Presidente Perón”, Buenos Aires, Argentina

Hospital General de Agudos "Dr. José María Ramos Mejía”, Ciudad Autónoma de Buenos Aires, Argentina

Hospital de Trauma y Emergencias "Dr. Federico Abete”, Buenos Aires, Argentina

Hospital de Niños “Sor María Ludovica”, La Plata, Buenos Aires, Argentina

Hospital del Trabajador, Santiago, Chile

Werner-Wicker-Klinik, Bad Wildungen, Alemania

Clínica Bazterrica, Ciudad Autónoma de Buenos Aires, Argentina

Hospital Italiano de Buenos Aires, Ciudad Autónoma de Buenos Aires, Argentina

Hospital Británico de Buenos Aires, Ciudad Autónoma de Buenos Aires, Argentina

Hospital Universitario La Paz, Madrid, España

Hospital Universitario Austral, Pilar, Buenos Aires, Argentina

Hospital for Special Surgery, Nueva York, Estados Unidos

Clínica del Deporte, La Plata, Buenos Aires, Argentina

Hospital Italiano de Buenos Aires, Ciudad Autónoma de Buenos Aires, Argentina

Hospital Universitario Austral, Pilar, Buenos Aires, Argentina

Hospital de Pediatría S.A.M.I.C. "Prof. Dr. Juan P. Garrahan”, Ciudad Autónoma de Buenos Aires, Argentina

Hospital Pediátrico Legaria, Ciudad de México, México

Hospital Alemán, Ciudad Autónoma de Buenos Aires, Argentina

Sanatorio de los Arcos, Ciudad Autónoma de Buenos Aires, Argentina

Grupo Médico Vertebral, Ciudad Autónoma de Buenos Aires, Argentina

Clínica La Pequeña Familia, Junín, Buenos Aires, Argentina

Hospital Italiano de Buenos Aires, Ciudad Autónoma de Buenos Aires, Argentina

Hospital Británico de Buenos Aires, Ciudad Autónoma de Buenos Aires, Argentina

Universidad del Valle, Cali, Colombia

Hospital Italiano de Buenos Aires, Ciudad Autónoma de Buenos Aires, Argentina

Facultad de Medicina, Universidad del Salvador, Ciudad Autónoma de Buenos Aires, Argentina

Hospital Provincial de Neuquén, Neuquén, Argentina

Sanatorio Allende, Córdoba, Argentina

Hospital Pediátrico “Dr. Avelino Lorenzo Castelán”, Chaco, Argentina

Hospital Nacional "Prof. Alejandro Posadas", Buenos Aires, Argentina

Hospital Santa Clara, Bogotá, Colombia

Banco de Prótesis, Montevideo, Uruguay

Cirugiadelpie.net, Ciudad Autónoma de Buenos Aires, Argentina

Sanatorio Allende, Córdoba, Argentina

Clínica Bazterrica, Ciudad Autónoma de Buenos Aires, Argentina

Hospital Universitario Quironsalud, Madrid, España

Hospital Británico de Buenos Aires, Ciudad Autónoma de Buenos Aires, Argentina

Instituto Cardiovascular de Buenos Aires (ICBA), Argentina

Hospital Italiano de Buenos Aires, Ciudad Autónoma de Buenos Aires, Argentina

Hospital Escuela de Agudos “Dr. Ramón Madariaga”, Posadas, Misiones 
Paganini, Federico

Pastrana, Martín J.

Patiño, Osvaldo J.

Pedro Maraude, Antonio E.

Pelaez, Carlos R.

Peratta, Marcela

Pereira, Enrique E.

Pereira, Sebastián

Pioli, Ignacio

Raimondi, Nicolás

Re, Rodrigo

Reboiras, Fabiana

Rellán, Ignacio

Remondino, Rodrigo

Restrepo Giraldo, José N.

Restrepo Noriega, Victoria E.

Reyes Sánchez, Alejandro A.

Río, Marcelo W.

Rodríguez, Javier L.

Romagnoli, Julián

Romanelli, Jorge M.

Roncoroni, Andrés

Rossi, Luciano A.

Sánchez, Ariel

Santamarta, Laura

Sanzana Edgardo S.

Schiappacasse, Carolina

Segura, Belisario

Sepúlveda Arriagada, Dalia

Sepúlveda Oviedo, Matías

Simesen de Bielke, Harold

Sirna, Pablo

Slullitel, Daniel

Slullitel, Pablo

Soria, Roberto D.

Sperone, Enzo D.

Stonski, Eduardo

Tello, Carlos A.

Teruya, Gustavo

Torrens Canova, Carlos

Valbuena, Sebastián E.
Sanatorio Allende, Córdoba, Argentina

Hospital de Pediatría S.A.M.I.C. "Prof. Dr. Juan P. Garrahan”, Ciudad Autónoma de Buenos Aires, Argentina

Hospital Italiano de Buenos Aires, Ciudad Autónoma de Buenos Aires, Argentina

Sanatorio Allende, Córdoba, Argentina

Hospital Interzonal General de Agudos “Gral. José de San Martín”, La Plata, Buenos Aires, Argentina

Centro Artro, Buenos Aires, Argentina

Hospital Universitario CEMIC, Ciudad Autónoma de Buenos Aires, Argentina

Hospital Sirio Libanés, Ciudad Autónoma de Buenos Aires, Argentina

Sanatorio Allende, Córdoba, Argentina

Hospital Universitario Austral, Pilar, Buenos Aires, Argentina

Sanatorio Allende, Córdoba, Argentina

Hospital Italiano de Buenos Aires, Ciudad Autónoma de Buenos Aires, Argentina

Hospital Italiano de Buenos Aires, Ciudad Autónoma de Buenos Aires, Argentina

Hospital de Pediatría S.A.M.I.C. "Prof. Dr. Juan P. Garrahan”, Ciudad Autónoma de Buenos Aires, Argentina

Hospital Universitario Departamental de Nariño, San Juan de Pasto, Nariño, Colombia

Clínica El Rosario, Medellín, Colombia

Instituto Nacional de Rehabilitación "Luis Guillermo Ibarra Ibarra", Ciudad de México, México

Clínica Zabala, Ciudad Autónoma de Buenos Aires, Argentina

Hospital General de Niños "Pedro de Elizalde”, Ciudad Autónoma de Buenos Aires, Argentina

Hospital General de Agudos “Dr. Juan A. Fernández”, Ciudad Autónoma de Buenos Aires, Argentina

Hospital General de Agudos "Dr. Juan A. Fernández”, Ciudad Autónoma de Buenos Aires, Argentina

Hospital de Pediatría S.A.M.I.C. "Prof. Dr. Juan P. Garrahan", Ciudad Autónoma de Buenos Aires, Argentina

Hospital Italiano de Buenos Aires, Ciudad Autónoma de Buenos Aires, Argentina

Centro de Endocrinología, Rosario, Santa Fe, Argentina

Hospital Central de San Isidro "Dr. Melchor Ángel Posse”, Buenos Aires, Argentina

Universidad de Concepción, Chile

Las Araucarias, Clínica de Rehabilitación, Buenos Aires, Argentina

Otamed, Ciudad, Mendoza, Argentina

COTI, Santiago, Chile

Hospital Base Valdivia, Chile

Sanatorio Modelo de San Miguel de Tucumán, Argentina

Centro de Estudio y Tratamiento Patología de Columna Vertebral Sustenta, Mar del Plata, Argentina

Sanatorio de la Mujer, Rosario, Santa Fe, Argentina

Hospital Italiano de Buenos Aires, Ciudad Autónoma de Buenos Aires, Argentina

Hospital Santa Isabel de Hungría, Guaymallén, Mendoza, Argentina

Hospital de Trauma y Emergencias “Dr. Federico Abete”, Buenos Aires, Argentina

Hospital Italiano de Buenos Aires, Ciudad Autónoma de Buenos Aires, Argentina

Hospital de Pediatría S.A.M.I.C. "Prof. Dr. Juan P. Garrahan”, Ciudad Autónoma de Buenos Aires, Argentina

Hospital Británico de Buenos Aires, Ciudad Autónoma de Buenos Aires, Argentina

Hospital Universitario del Mar, Barcelona, España

Hospital El Cruce, Universidad Nacional Arturo Jauretche, Florencio Varela, Buenos Aires, Argentina 
Varaona, José M.

Vedoya, Santiago P.

Vega Mayer, Rafael

Vernengo Lezica, Alejo

Vindver, Gabriel

Viollaz, Gonzalo M.

Yurac, Ratko

Zaidenberg, Ezequiel E.

Zancolli, Pablo
Hospital Alemán, Ciudad Autónoma de Buenos Aires, Argentina

Hospital Británico de Buenos Aires, Ciudad Autónoma de Buenos Aires, Argentina

Clínica San Carlos de Apoquindo, Santiago, Chile

Hospital Central de San Isidro "Dr. Melchor Ángel Posse”, Buenos Aires, Argentina

Hospital Sirio Libanés, Ciudad Autónoma de Buenos Aires, Argentina

Centro Artro, Ciudad Autónoma de Buenos Aires, Argentina

Clínica Alemana de Santiago, Santiago, Chile

Hospital Italiano de Buenos Aires, Ciudad Autónoma de Buenos Aires, Argentina

Fundación Favaloro, Ciudad Autónoma de Buenos Aires, Argentina 